\title{
ENFERMEDAD DE BEHÇET EN UN PACIENTE CON LUPUS ERITEMATOSO SISTÉMICO, REPORTE DE UN CASO CLÍNICO
}

\author{
López Bravo Marcela Tatiana ${ }^{1}$, Román Zambrano María del Cisne ${ }^{2}$, \\ Mendieta Maza Víctor Daniel ${ }^{3}$, Peña Armijos Madeleyne Victoria ${ }^{4}$.

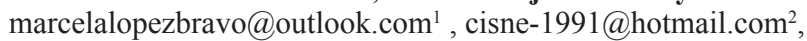 \\ vic_daniel1990@hotmail.com ${ }^{3}$ madeley2823@gmail.com ${ }^{4}$ \\ https://orcid.org/0000-0002-0777-76941, https://orcid.org/0000-0002-1045-40012 \\ https://orcid.org/0000-0002-2969-9586 ${ }^{3}$, https://orcid.org/0000-0002-3174-1800 ${ }^{4}$ \\ IESS Hospital General Machala, Hospital de Espacialidades Carlos Andrade Marín. \\ Machala-Ecuador
}

Recibido (07/08/20), Aceptado (21/08/20)

\begin{abstract}
Resumen: La enfermedad de Behçet (EB) es una vasculitis mulsistémica, fue descrita como un complejo trisintomático de úlceras orales, úlceras genitales e iritis de carácter recidivante, que afecta principalmente a adultos jóvenes. Muchas de sus manifestaciones clínicas pueden ser autolimitadas. A continuación presentamos el caso clínico de un paciente femenino de 35 años con antecedentes patológicos personales de lupus eritematoso sistémico, que presentó lesiones úlceras aftosas en mucosa oral, úlceras genitales y úlceras cutáneas, además de congestión conjuntival. El objetivo de la presente publicación es aportar un caso clínico de Enfermedad Behçet con manifestaciones dermatológicas características de la enfermedad, lo cual constituye un reto diagnóstico para una patología poco frecuente.
\end{abstract}

Palabras Clave: Enfermedad de Behçet, vasculitis

\section{BEHCET'S DISEASE IN A PATIENT WITH SYSTEMIC LUPUS ERYTHEMATOSUS, A CASE REPORT}

\begin{abstract}
Behçet's disease (EB) is a mulsystemic vasculitis, it was described as a trisymptomatic complex of oral ulcers, genital ulcers and recurrent iritis, which mainly affects young adults. Many of its clinical manifestations can be self-limiting. Below we present the clinical case of a 35-year-old female patient with a personal pathological history of systemic lupus erythematosus, who presented with aphthous ulcers on the oral mucosa, genital ulcers, and skin ulcers, in addition to conjunctival congestion. The objective of this publication is to provide a clinical case of Behçet disease with characteristic dermatological manifestations of the disease, which constitutes a diagnostic challenge for a rare pathology.
\end{abstract}

Keywords: Behçet's disease, vasculitis. 


\section{I.INTRODUCCIÓN}

El síndrome de Behçet o Enfermedad de Behçet (EB) se caracteriza por úlceras aftosas recurrentes orales y/o genitales, así como por afectación cutánea, ocular, articular, gastrointestinal y/o del sistema nervioso central [1]. La vasculitis que ocurre puede afectar arterias o venas de cualquier tamaño. Se desconoce la causa subyacente del síndrome de Behçet. Al igual que con otras enfermedades autoinmunes, el trastorno puede representar una actividad inmune aberrante desencadenada por la exposición a un agente, quizás infeccioso, en pacientes con una predisposición genética a desarrollar la enfermedad. Se la considera una enfermedad utoinmune, ya que la principal lesión anatomopatológica es una vasculitis, que parece estar ligada al aloantígeno HLA-B5 (B51) [2].

Además la EB ocasiona lesiones inflamatorias en múltiples órganos como la piel, las articulaciones y en los sistemas gastrointestinal, renal, cardiopulmonar y/o neurológico [3].

$\mathrm{Su}$ espectro clínico se ha ampliado considerablemente e implica a numerosos órganos y sistemas. Puede aparecer en individuos de todas las edades con predominio en varones entre la $3^{\mathrm{a}}$ y $4^{\mathrm{a}}$ década de la vida [2].

La prevalencia varía de 13.5 a 20 por 100,000 en Japón, Corea, China, Irán y Arabia Saudita. Por ser una enfermedad de difícil diagnóstico su prevalencia es difícil de estimar. Es algo más común en hombres en el área del mediterráneo oriental y en mujeres en países del norte de Europa. [4].

Los agentes desencadenantes propuestos incluyen antígenos virales y bacterianos u otras fuentes ambientales, como productos químicos o metales pesados. El estudio histopatológico y los datos analíticos son inespecíficos, su diagnóstico se basa en el método clínico: signos y síntomas (descripción y agrupación), para lo cual se han diseñado múltiples criterios de diagnóstico según la predominancia sintomática [5].

El diagnóstico de la EB se realiza en base a criterios clínicos predefinidos según distintos grupos de estudio. Dentro de éstos, los más utilizados son los Criterios Internacionales para el diagnóstico de la Enfermedad de Behcet, (ICBD, por sus siglas en inglés), y el grupo internacional de estudio de la enfermedad de Behcet (ISG) [6]. La mayoría de los pacientes pueden clasificarse de acuerdo a los criterios del ISG creados en 1990, los cuales poseen una excelente especificidad, pero carecen de sensibilidad, por lo que fueron modificados en el 2006, formando: Criterios Internacionales para el diagnóstico de la Enfermedad de Behcet (ICBD). En esa última revisión fueron incluidas las lesiones vasculares (trombosis arteriales, venosas, aneurismas) como criterio diagnóstico [7].

A continuación presentamos el siguiente caso clínico fue reportado en el Hospital General IESS Machala.

\section{II.DESARROLLO}

Paciente femenino de 35 años de edad con antecedentes patológicos personales de LES (Lupus Eritematoso Sistémico) desde hace 7 años en tratamiento: Rituximab, Medrol e Hidroxicloroquina, se mantenía sin actividad reciente de LES y sin antecedentes previos de compromiso renal. Ingresó a nuestra unidad hospitalaria en mes de junio del 2020, por presentar desde hace 10 días lesiones cutáneas de aspecto vesiculoso con base eritematosa, que tienden a la ulceración, en tronco (región submamaria) y región genital, de forma y tamaño variable, se acompaña de dolor intenso en dichas lesiones. Además paciente refiere que desde hace varios meses presenta úlceras aftosas en mucosa oral y desde hace un mes presentó conjuntivas eritematosas, actualmente ha disminuido la congestión ocular.

Durante la exploración física se evidenció marcado compromiso del estado general del paciente. La exploración oftalmológica se encontró dentro de parámetros normales tanto en segmento anterior como en el fondo de ojo. Cavidad oral se evidenció: lesiones aftosas, de color rojizo, de forma ovalada, muy dolorosas, de aproximadamente $20 \mathrm{~mm}$, en mucosa oral, borde de lengua y paladar blando, dificultando la deglución, ver fig. 1 . A nivel de tórax en región infra mamaria presenta en piel lesiones ulcerosas profundas, con base eritematosa y centro cubierto de fibrina amarillenta, ver fig. 2. En la exploración inguinogenital: Región vulvar: se observan varias úlceras profundas de tamaño variable 2 a $3 \mathrm{~cm}$, la mayoría de ellas con centro amarillento y otras con bordes y centros necróticos, ver fig. 3 .

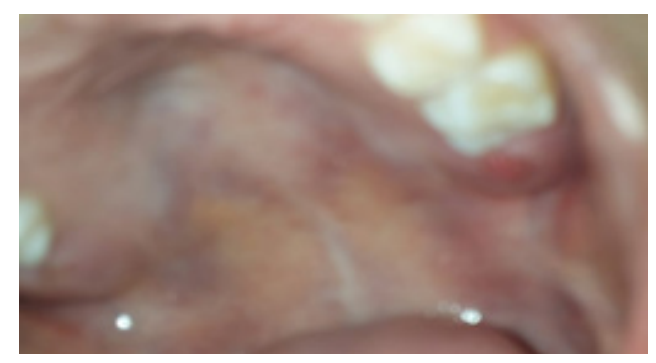

Fig 1. Lesiones ulcerosas rojizas en mucosa oral 


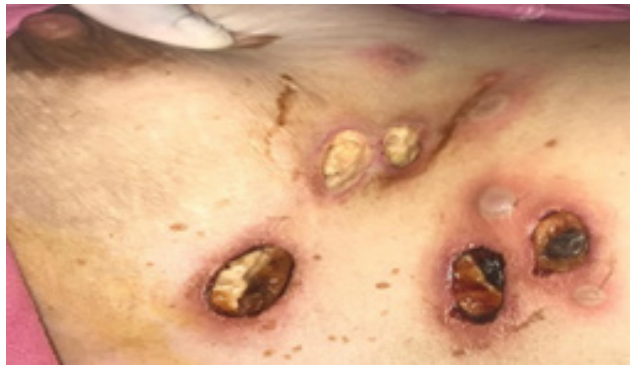

Fig 2. Lesiones cutáneas ulcerosas profundas, centro con base de fibrina amarillenta, en región submamaria.

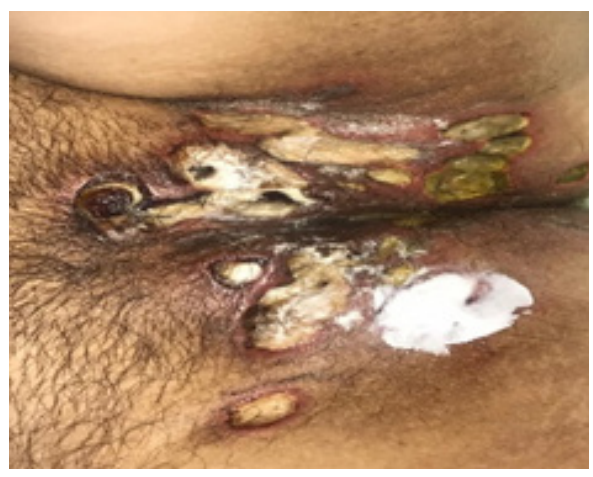

Fig 3. Lesiones genitales ulceradas profundas amarillentas y necróticas.

\section{A.Laboratorio}

TABLA I. Biometría hemática, química sanguínea

\begin{tabular}{ll}
\hline & Resultados \\
\hline Leucocitos & 3.49 \\
Linfocitos & $25.35 \%$ \\
Monocitos & $11.5 \%$ \\
Basófilos & 1.35 \\
Glóbulos rojos & 3.1 \\
HGB g/dl & 10.1 \\
HCT & $30.2 \%$ \\
MCV fl & 86.2 \\
MCH pg & 28.8 \\
MCHC g/dl & 33.4 \\
VSG mm/h & 41 \\
Glucosa mg/dl & 117.70 \\
NA mEq/l & 134 \\
K mEq/l & 4.20 \\
Urea mg/dl & 166 \\
Creatinina mg/dl & 2.46 \\
Ácido úrico mg/dl & 1.7 \\
Albúmina g/dl & 1.7 \\
Globulina g/dl & 1.8 \\
Proteínas totales g/dl & 3.5 \\
Ferritina & 1595 \\
Dimero D & 749.48 \\
Plaquetas x 1000/ul & 46 \\
Nutrófilos & $61.8 \%$ \\
LDH & 189 \\
PCR & 435 \\
Procalcitonina & 12.64 \\
T GO & 9 \\
T GP & 18 \\
\hline & \\
&
\end{tabular}

En las pruebas laboratorio (biometría hemática, química sanguínea) se observó: pancitopenia, hipoproteinemia, elevación de azoados, elevación de reactantes de fase aguda, ver tabla I. El EMO: no infeccioso y proteinuria en 24 horas negativa.

Además las pruebas inmunológicas: anticuerpos antinucleares ANA, anti-DNA, anti RO/SS-A, anti SM, ANCA C, ANCA P, Anti Coagulante lúpico, IgM anticardiolipina, IgG anticardiolipina, todas resultaron negativas. HIV No reactivo.

\section{B.Exámenes de imagenología}

Ultrasonido de abdomen superior y Pélvico: Estudio Normal, sin patología.

TAC simple de tórax: Estudio normal, sin patología.

\section{C.Cultivos}

Cultivo de herida en Región genital: rescate de Pseudomona Aeruginosa KPC positivo

\section{D.Histopatológico}

Tejido: piel de región inguinal derecha.

Descripción microscópica: Los cortes histológicos corresponden a piel tapizada por epitelio escamoso estratificado en uno de los bordes ulcerado rodeado por tejido necrosado y elementos celulares inflamatorios mixtos, a nivel de la dermis se observa vasos sanguíneos de pequeño calibre rodeados por elementos celulares inflamatorios de predominio mononucleares. Conclusión: Hallazgos histológicos compatibles con vasculitis de pequeños vasos. Ulceración inespecífica.

Paciente permaneció larga estancia hospitalaria, con diagnóstico de ingreso de sepsis de partes blandas SOFA 2 puntos, Enfermedad Renal Aguda AKIN III, con requerimiento de terapia de sustitución renal. Además por la localización y características de las lesiones aftosas en mucosa oral y por las úlceras vaginales y cutáneas, se llegó al diagnóstico sugestivo de Enfermedad de Behçet que se corroboró con la biopsia de tejido reportando: vasculitis de pequeños vasos. Se instauró al tratamiento, corticoide sistémico, inmunosupresores (azatriopina y talidomida), colchicina, además de antibióticoticoterapia de amplio espectro por bacteria multiresistente aislada en el cultivo de herida.

Durante la cuarta semana de hospitalización, paciente con evidente mejoría clínica, proceso infeccioso de partes blandas en recuperación, en los estudios paraclínicos se evidenció mejoría en el recuentro leucocitario y plaquetario, azoados en parámetros normales, por lo que se suspendió la terapia sustitutiva renal. Además las lesiones cutáneas y las úlceras en mucosa oral y vagi- 
nal, se encontraban en mejoría clínica, en proceso de esfacelamiento con eliminación de fibrina, con nula existencia de material necrótico y purulento.

A la semana de su hospitalización. Ver fig. 4, fig. 5.

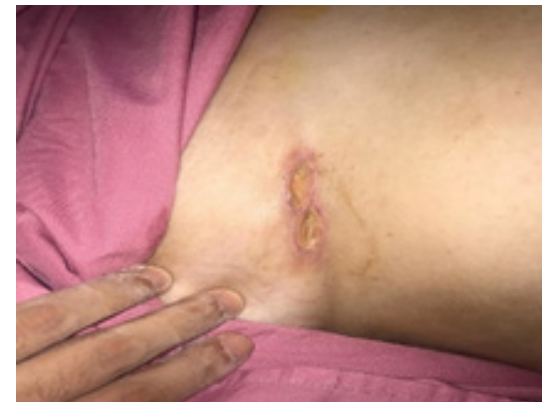

Fig 4. Lesiones ulcerosas submamaria en proceso de cicatrización

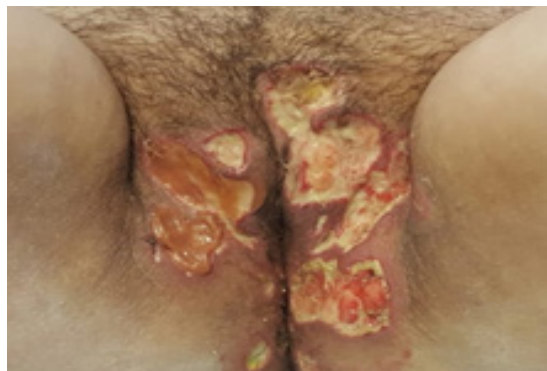

Fig 5. Lesiones ulcerosas en región genital con tejido de cicatrización

A la 4ta semana de hospitalización. Ver fig 6, fig 7.

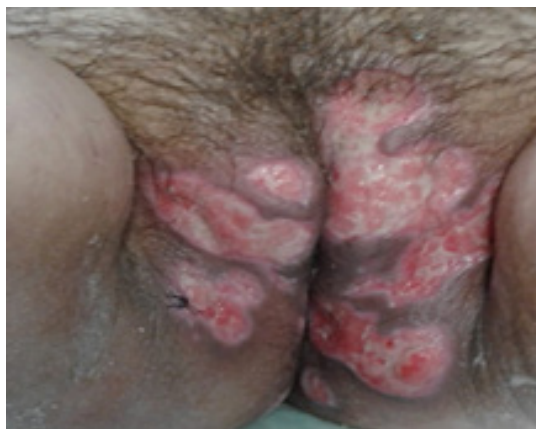

Fig 6. Región genital úlceras en labios mayores en proceso de cicatrización.

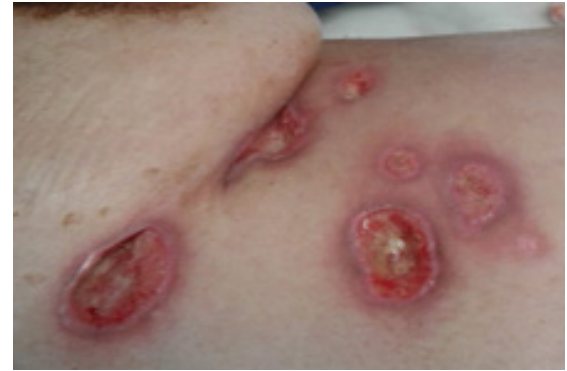

Fig 7. Región subamamaria, úlceras en proceso de cicatrización

\section{III.METODOLOGÍA}

La enfermedad de Behçet (BD) es una enfermedad vascular inflamatoria crónica recurrente sin prueba patognomónica [8].

El diagnóstico se basa en la asociación de aftosis bipolar, manifestaciones cutáneas, uveítis, sobre todo posterior y afectación de grandes vasos [9]. En ausencia de estos signos es más difícil confirmar el diagnóstico. No existen pruebas diagnósticas que confirmen su presencia, por lo que su diagnóstico es clínico. El grupo internacional de estudio de la enfermedad de Behcet (ISG) definió criterios clínicos para el diagnóstico de la enfermedad, algunos de ellos cumplía la paciente del caso clínico como son: las lesiones ulcerosas de bordes definidos, rodeados de halo eritematoso, y fondo amarillento, tanto en mucosa oral, genital y región infra mamaria. Aplicando los Criterios Internacionales para el diagnóstico de la Enfermedad de Behcet, (ICBD, por sus siglas en inglés) dieron como resultado 5 puntos (lesiones aftosas orales, lesiones ulcerosas genitales, y las úlceras en piel), de acuerdo a estos criterios un paciente con una puntuación igual o mayor a 4 puntos se clasifica como Enfermedad de Behçet [6]. Sin embargo, hay que realizar diagnósticos diferenciales en dependencia de las manifestaciones clínicas que se presenten en diversas enfermedades como la estomatitis aftosa recurrentes, infecciones virales (herpes simple, citomegalovirus, Epstein Barr, sífilis), síndrome de Reiter, esclerosis múltiple, entre otras [10]. Para lo cual se realizó estudio histopatológico, encontrándose: tejido necrosado y elementos celulares inflamatorios mixtos, a nivel de la dermis se observa vasos sanguíneos de pequeño calibre rodeados por elementos celulares inflamatorios de predominio mononuclear, compatible con vasculitis de pequeños vasos.

\section{IV.RESULTADOS}

La enfermedad de Behcet es una enfermedad vas- 
cular inflamatoria crónica, sin signos característicos, de etiología desconocida y de difícil diagnóstico. Las manifestaciones clínicas del paciente constituyen la base del diagnóstico, los criterios fueron modificados en el 2006 por el grupo internacional para el estudio de Enfermedad de Behcet (ICBD) Según las características de las lesiones orales, genitales y en piel que presentó la paciente se clasifica como enfermedad de Behcet $(5$ puntos) ver tabla II, sumado a ello el estudio histopatológico de la biopsia piel de región inguinal, la misma que revela infiltrados perivasculares con células inflamatorias mononucleares, compatibles con la vasculitis oclusiva mediada por mecanismos inmunes, presentes en la mayor parte de casos en pacientes con Enfermedad de Behcet [5].

TABLA II. Criterios Internacionales Enfermedad de Behçet (ICBD). Puntuación $\geq 4$ indica diagnóstico.

\begin{tabular}{lc}
\hline Signos/síntomas & Puntos \\
\hline Lesiones oculares & 2 \\
Aftas genitales & 2 \\
Aftas orales & 2 \\
Lesiones en piel & 1 \\
Manifestaciones neurológicas & 1 \\
Manifestaciones vasculares & 1 \\
Patergia positivo & 1 \\
\hline El test de patergia es opcional y el sistema clasificatorio \\
original no lo incluye. Sin embargo, si es realizado, un punto \\
adicional se asignará a los resultados. \\
\multicolumn{2}{|l}{ Adaptado de: The International Criteria for Behçet's Disease } \\
(ICBD): a collaborative study of 27 countries on the \\
sensitivity and specificity of the new criteria.[7] \\
\hline
\end{tabular}

vascular inflamatoria crónica, sin signos característicos de la enfermedad, de etiología desconocida y de difícil diagnóstico. Las manifestaciones clínicas del paciente constituyen la base del diagnóstico, los criterios diagnósticos fueron modificados en el 2006 por el grupo internacional para el estudio de Enfermedad de Behcet (ICBD) Según las características de las lesiones orales, genitales y en piel que presentó la paciente se clasifica como enfermedad de Behcet (5 puntos) ver tabla II, sumado a ello el estudio histopatológico de la biopsia piel de región inguinal, la misma que revela infiltrados perivasculares con células inflamatorias mononucleares, compatibles con la vasculitis oclusiva mediada por mecanismos inmunes, presentes en la mayor parte de casos en pacientes con Enfermedad de Behcet [5].

\section{V.CONCLUSIONES}

1.La enfermedad de Behçet que inicialmente se describió como un cuadro autoinmune, es un proceso auto inflamatorio crónico, de baja frecuencia y de etiología desconocida, generalmente se caracteriza por presentar úlceras orales, genitales e iritis, estas úlceras son muy dolorosas, ante un cuadro clínico que presente estas características se debe considerar el diagnóstico probable de Enfermedad de Behçet, ya que sin un tratamiento específico inmediato podría incrementar su morbimortalidad [11].

2.Ante un diagnóstico probable Enfermedad de Behçet se usa actualmente los Criterios Internacionales de Enfermedad de Behçet (ICBD), modificadas en el 2006, que presentan una sensibilidad mayor que el Grupo Internacional para el estudio de enfermedad de Behcet (ISG) creados en 1990 [6].

3.El tratamiento depende de las manifestaciones clínicas. La lesiones mucocutáneas responden en general al tratamiento con corticoides tópicos. La colchicina, resulta útil debido a su acción inhibitoria sobre la función de los neutrófilos. La talidomida ha demostrado ser muy eficaz en la resolución de las lesiones mucocutáneas refractarias a otros tratamientos. Su mecanismo de acción es a través de la inhibición del TNF- $\alpha$ y de la angiogénesis. En casos de enfermedad mucocutánea grave están indicados los corticoides sistémicos [7].

Este estudio concluyó que luego del uso de corticoides sistémicos, inmunosupresores, hubo mejoría notable de las lesiones en piel, de ahí que la instauración inmediata al tratamiento en función a las manifestaciones clínicas de Enfermedad de Behçet constituyen de mucha importancia. 


\section{REFERENCIAS}

[1]J. Calvo Catalá, A. Baixauli Rubio, C. Campos Fernández y M. I. González-Cruz Cervellera, «Enfermedad de Behçet,» Revista de la SVR Sociedad Valenciana de Reumatología, pp. 313-330, 2015.

[2]L. C. Pérez, «Enfermedad de Behçet,» An. Med. Interna (Madrid), vol. 8, 2001.

[3]R. Molina, A. Huerta-Rosario, C. Alva, K. Mejía, N. Mori y R. Romero, «Enfermedad de neuro-Behçet en Perú: reporte de caso y revisión de la literatura,» Revista Biomédica Revisada Por Pares, vol. 1, no 1, p. 1, 2017.

[4]. S. Ellison L y Y. Yusuf, «Patogenia del síndrome de Behçet,» UpToDate, 2020.

[5]W. Castillo González, J. González-Argote y J. Hernández Estévez, «Behcet's disease,» Cuba Reumatol, Septiembre 2014.

[6]F. Davatchi, S. Assaad-Khalil, K. Calamia, B. Sadeghi-Abdollahi, M. Schirmer y C. C. Zouboulis, «The International Criteria for Behçet's Disease (ICBD): A collaborative study of 27 countries on the sensitivity and specificity of the new criteria,» Journal of the European Academy of Dermatology and Venereology, pp. 338-347, 2014.

[7]W. González, J. González-Argote y E. J. Hernández, « Enfermedad de Behçet Enfermedad de Behçet Behcet's disease,» Revista Cubana de Reumatología, vol. 3, pp. 309-321, 2014.

[8]J. E. Acad, «The International Criteria for Behçet's Disease (ICBD): a collaborative study of 27 countries on the sensitivity and specificity of the new criteria,» JEADV, pp. 338-347, 2013.

[9]M. R. Carballeira y G. Espinosa Garriga , «MONOGRÁFICO: Enfermedades de Behçet,» Cuadernos de Autoinmunidad, pp. 4-6, 2011. [10]M. E. Alfonso Valdés, «Síndrome de Behcet ARTÍCULO,» Revista Cubana de Hematologia, Inmunologia y Hemoterapia, vol. 36, pp. 301-315, 2016.

[11]S. D, B. Wechsler y K. Desseaux , «Mortality in Behçet's disease,» Arthritis Rheum., p. 2806-2812, 2010.

\section{RESUMEN CURRICULAR}

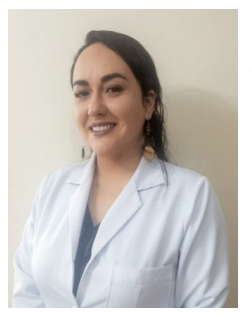

Marcela López, Médico General, otorgado, por la Universidad Tecnológica Equinoccial, Quito-Ecuador, actualmente cursa residencia médica en el área de medicina interna, en el Hospital General IESS Machala.

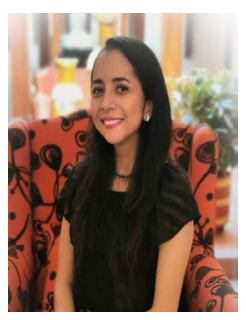

María del Cisne Román, Médico General, otorgado por la Universidad Técnica de Machala, Machala-Ecuador, actualmente cursa residencia médica en el área de medicina interna, en el Hospital General IESS Machala.

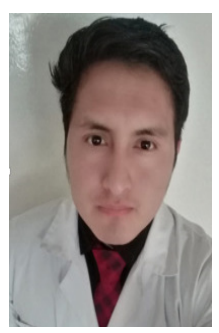

Daniel Mendieta, Médico General, otorgado, por La Escuela Superior Politécnica del Chimborazo, RiobambaEcuador, actualmente cursa residencia Médica en el Área Covid 3, en el Hospital de Especialidades Carlos Andrade Marín.

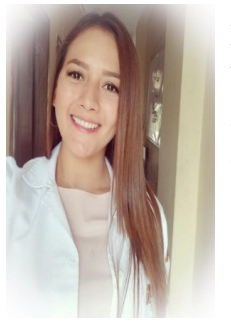

Madeleyne Peña, Médico General, otorgado por la Universidad Católica de Cuenca, Cuenca-Ecuador, actualmente cursa residencia médica en el Área Covid 1, en el Hospital General IESS Machala. 